\title{
AUTOPERCEPCIÓN DE LA LABOR DOCENTE UNIVERSITARIA: IDENTIFICANDO COMPETENCIAS INVESTIGATIVAS APLICABLES EN EL 2020
}

\section{UNIVERSITY TEACHER'S WORK SELF-PERCEPTION: IDENTIFYING APPLICABLE RESEARCH COMPETENCES IN 2020}

\section{Guillermo Alexander Quezada Castro'}

María del Pilar Castro Arellano²

Juan Manuel Oliva Núñez ${ }^{3}$

María del Pilar Quezada Castro ${ }^{4}$

Universidad Alas Peruanas, filial Piura - Perú

\section{RESUMEN}

La labor docente en las universidades se caracteriza por las competencias investigativas básicas, complementarias y avanzadas, las

\footnotetext{
1 Abogado, Maestro en Derecho Civil y docente a
} tiempo completo en la Universidad Alas Peruanas, filial Piura $\square$ Perú. Correo electrónico: g quezada_ca@doc.uap.edu.pe 2 Abogada, Licenciada en Trabajo Social, Maestro en Derecho Civil, Doctora en Derecho, Coordinadora de la Escuela Profesional de Turismo, Hotelería y Gastronomía de la Universidad Alas Peruanas, filial Piura - Perú. Correo electrónico: $m$ castro@uap.edu.pe

3 Arqueólogo, Doctor en Ciencias de la Educación y docente a tiempo completo en la Universidad Alas Peruanas, filial Piura - Perú. Correo electrónico:j_oliva@doc.uap.edu.pe $4 \quad$ Abogada, Maestro en Derecho Civil y docente a tiempo completo en la Universidad Alas Peruanas, filial Piura $\square$ Perú. Correo electrónico: m quezada ca@doc.uap.edu. pe cuales consolidan la relación con el estudiante en su proceso de formación académica. En la presente investigación se planteó como objetivo "identificar las competencias investigativas del docente universitario a partir de su autopercepción". Para ello se desarrolló el enfoque cuantitativo, de tipo descriptivo, con diseño no experimental, método analítico, técnica de la encuesta e instrumento - cuestionario cerrado, el cual se realizó a 36 profesionales, en su labor como docentes en las universidades de la Región Piura - Perú. Como principal resultado se obtuvo que la participación activa del docente universitario involucra la difusión o divulgación de la investigación en revistas científicas, 
concluyéndose que sí existe autopercepción de la labor docente en su rol como investigador en la universidad.

PALABRAS CLAVE: Autopercepción, competencias investigativas, docente.

\section{ABSTRACT}

The teaching work in universities is characterized by basic, complementary and advanced research skills, which consolidate the relationship with the student in their academic training process. In the present investigation, the objective was "to identify the research competencies of the university teacher based on their self-perception", for this purpose the quantitative approach was developed, of a descriptive type, with a non-experimental design, analytical method, survey technique and instrument - closed questionnaire, which was conducted to 36 professionals, in their work as teachers in the universities of the Piura Region - Peru. As a main result, it was obtained that the active participation of the university teacher involves the dissemination or dissemination of research in scientific journals, concluding that there is self-perception of the teaching work in its role as a researcher in the university.

KEYWORDS: Self-perception, research skills, teacher.

\section{INTRODUCCIÓN}

La relación docente - estudiante es vital para el sostenimiento dinámico, transversal y actualizado de cada asignatura, constituye el elemento diferenciador en el aprendizaje universitario, toda vez que está prediseñada para superar el ámbito de la lectura, a través del respectivo análisis y síntesis de un determinado tema, situación que debería extenderse a la creación de contenidos propios, labor pasible de ser promovida desde los primeros ciclos de formación académica.
El dinamismo en aula permite a los actores mencionados la participación activa, relacionada con la identificación de sus roles e interiorización de la sesión temática correspondiente, facilitando la intervención natural, desinteresada y espontánea hacia las demás asignaturas.

El desarrollo transversal de las asignaturas permite concebir la multidisciplinariedad de cada eje temático, de esta forma, se difunde y ejemplifica las percepciones que versan respecto a un mismo hecho o acontecimiento, integrándolo holísticamente al desenlace verificable en la sociedad.

La actualización de los conocimientos impartidos en aula, no debe estar encaminada a la repetición de conocimientos, al contrario, debe ser parte del aprendizaje continuo, generándose la propia versión de lo aprendido.

En ese orden de ideas, surge la curiosidad del equipo investigador, ¿cuál es la autopercepción del docente universitario en su rol de investigador?, para lo cual se requiere el objetivo: "identificar las competencias investigativas del docente universitario a partir de su autopercepción".

\section{DESARROLLO}

La investigación está relacionada con la producción científica, la misma que debe ser promovida en toda casa de estudios superiores, es por ello que según López, Márquez \& Alonso (1999) citados en Tovar-Gálvez (2018) señalaron que es importante "comprender la enseñanza y el aprendizaje como un proceso de interacciones", las cuales se fomentan en el ámbito educativo (Veytia, 2012), comprobándose en la formación de los profesionales en el oficio investigativo (Rojas \& Aguirre, 2015), desde su etapa inicial como estudiantes (Rivera, 2017), lo cual permitirá "crear e innovar productos y servicios" (Pulido, 2017), porque "la universidad se define 
desde el siglo XIX como un espacio donde se producen conocimientos" (Balbo, Pacheco \& Rangel, 2015) y se ejecutan procesos de investigación como lo precisa la sociedad actual (Reiban, 2018).

La investigación docente está relacionada con la resolución de problemas, según Ermeling (2010) citados en Nakamura, Rivero \& Velasco (2019); para lograr ello, resulta imprescindible identificar el perfil del profesor universitario (Mas, 2011), siendo la universidad "el espacio propicio para la formación de investigadores educativos" (Benitorevollo, Olier, Medrano \& González, 2016), puesto que "la Educación Superior juega un papel estratégico en la formación de las nuevas generaciones que afrontarán los retos de la globalización y de la era del conocimiento" (Martínez, A. et al., 2015), atender a lo descrito, permitirá mejorar la calidad en la educación (Toro, Saldarriaga, León, Martínez \& Arias, 2014).

La educación superior está asociada al concepto de competencia investigativa, según Montes de Oca \& Machado (2009); Estrada (2014); Mas-Torelló (2016) citados en Rubio, Torrado, Quirós \& Valls (2018), su desarrollo permitirá identificar que "el investigador sea novel o experimentado" (Marcano, 2010, citado en Reiban, De la Rosa \& Zeballos, 2017), es decir, deben primar "las actitudes, habilidades $y$ destrezas necesarias para llevar a cabo la elaboración de un trabajo de investigación" (Martínez \& Borjas, 2015, citados por Parra, Marsollier \& Difabio, 2019), debiendo tener presente que "sólo hay ciencias epistemológicamente definidas que indagan e intentan comprender la realidad del modo más riguroso posible" (Gayol, Montenegro, Tarrés \& D'Octavio, 2008), por lo tanto, "el docente enseña a investigar" (Arrieta, Ocón, López, Buelvas \& Monterroza, 2018).

En ese orden de ideas, surge el investigador, el cual según Delgado \& Alfonzo
(2019) "es aquella persona denominada sujeto-investigador que, apoyado en su intencionalidad, curiosidad y criticidad comienza a buscar conocer, para compensar necesidades particulares, mutuas, materiales, científicas, en cuyo proceso va adquiriendo, acumulando nociones y prácticas", el mismo que debe reunir capacidades y actitudes del docente (Borjas, 2000, citado en Aular, Marcano \& Moronta, 2009), por lo tanto, "debe brindar a todos educandos, conocimientos de carácter global y una formación de valor universal" (Chacín, 2018), porque la acción investigativa estimula la construcción permanente de las formas de organización (Maldonado \& et. al, 2007, citados en Borroto, Dueñas \& Reyna, 2018).

El docente debe ejercer su papel de formador integral (Ricardo, Amat, Andrade, Jiménez \& Cisneros, 2019); sin embargo, para conseguir esta meta, se requiere la eliminación de las prácticas tradicionales y estrictas (Rodas, 2015, citado en Zúñiga \& Pando, 2019) que originan la deficiente calidad académica de algunas instituciones (Pérez, 2012), es por tal motivo, que se requiere "nuevos líderes científicos entre profesores" (Ponce, Pupo, Ponce, Viteri \& Álvarez, 2017), quienes tienen el deber de inculcar en sus estudiantes el pensamiento crítico y creatividad (Marrero \& Pérez, 2014), contexto que impulsará la formación en metodologías (Martín, 2012, citado en Tapia, Cardona \& Vázquez, 2018) para desarrollar una investigación educativa (Buendía-Arias, Zambrano-Castillo \& Alirio, 2018) e impulsar "la generación de capacidades investigativas e innovativas en la educación superior" (Gómez, Aranda \& Santos, 2017; Herrera \& Didriksson, 1999, citados en Cardoso \& Cerecedo, 2018).

En este contexto, es necesario resaltar que según Araujo (2016) "las competencias se conciben como una metodología en la cual se pondera el saber hacer", surgiendo la necesidad 
de dar un enfoque curricular centrado en los requerimientos sociales modernos (Delors, 1996, citado Paz \& Fierro, 2015), generando una cultura de investigación (Bondarenko, 2009, citado en Véliz \& Lovato, 2019), capaz de solucionar los problemas inherentes al objeto de su profesión (Cruzata-Martínez, Bellido, Velásquez-Tejeda \& Alhuay-Quispe, 2018), adecuado a cada institución educativa (Campos, Madriz, Brenes, Rivera \& Viales, 2012), con la garantía de implementar proyectos de investigación e innovación dentros sus áreas disciplinarias (Mena \& Lizenberg, 2013, citado en Reiban \& Vera, 2017).

La labor docente se debe impartir desde la formación de competencias integrales investigativas que permitan una educación científica-cultural (Marrero \& Pérez, 2014, citados en Ayala \& Barrera, 2018), con lo cual se evidencia "un conjunto de habilidades, destrezas, y conocimientos" (García, 2019), las cuales se comprueban en el proceso de enseñanza - aprendizaje (Paz, Estrada, Chinchilla \& Valladares, 2017), a través del diálogo, reflexión y crítica (Aldana, 2017), clasificándose en "competencias conceptuales, procedimentales y actitudinales" (Guity \& Mendoza, 2018), apreciándose un carácter holístico, dinámico, evolutivo y ético (Aznar, Cáceres \& Hinojo, 2011, citados en García-Gutiérrez \& AznarDíaz, 2019), manifestándose a través de la productividad, calidad o innovación (Ollarves \& Salguero, 2009).

\section{METODOLOGÍA}

El enfoque de investigación fue cuantitativo, de tipo descriptivo, con diseño no experimental, método analítico, técnica de la encuesta e instrumento - cuestionario cerrado; el cual se realizó a 36 profesionales, en su labor como docentes en las universidades de la Región Piura - Perú, quienes participaron de manera voluntaria.

El equipo investigador consideró conveniente segmentar las competencias investigativas, asignándoles valores: 1 (nunca), 2 (casi nunca), $\mathbf{3}$ (a veces), $\mathbf{4}$ (casi siempre) y $\mathbf{5}$ (siempre), conforme se aprecia a continuación:

\section{a) Competencias básicas}

\begin{tabular}{|c|c|c|c|c|c|c|}
\hline \multicolumn{2}{|r|}{ Instrumento } & 1 & 2 & 3 & 4 & 5 \\
\hline P1 & $\begin{array}{l}\text { En el proyecto de tesis, ¿se debe considerar "paradigma, enfoque y } \\
\text { método"? }\end{array}$ & & & & & \\
\hline P2 & ¿Considera que el título es importante en el proyecto de tesis? & & & & & \\
\hline P3 & $\begin{array}{l}\text { Para elaborar un proyecto de tesis ¿se requiere "seguir instrucciones } \\
\text { de un solo autor? }\end{array}$ & & & & & \\
\hline P4 & $\begin{array}{l}\text { ¿Es necesario considerar las líneas de investigación al elaborar un } \\
\text { proyecto de tesis? }\end{array}$ & & & & & \\
\hline P5 & $\begin{array}{l}\text { En el último trimestre ¿ha recibido cursos de especialización } \\
\text { relacionados con metodología de la investigación científica? }\end{array}$ & & & & & \\
\hline P6 & ¿EI diseño metodológico es la esencia en un proyecto de tesis? & & & & & \\
\hline P7 & ¿Es necesario curso (s) de actualización en redacción cientifica? & & & & & \\
\hline P8 & $\begin{array}{l}\text { ¿Es necesario curso (s) de actualización en estadística aplicada a la } \\
\text { investigación? }\end{array}$ & & & & & \\
\hline P9 & $\begin{array}{l}\text { ¿Utiliza gestores de referencias bibliográficas (Zotero, Mendeley, } \\
\text { entre otros) en el proyecto de tesis? }\end{array}$ & & & & & \\
\hline P10 & ¿Recomienda "temas de investigación" a sus estudiantes? & & & & & \\
\hline
\end{tabular}


b) Competencias complementarias

\begin{tabular}{|c|c|c|c|c|c|c|}
\hline \multicolumn{2}{|r|}{$\begin{array}{l}\text { Instrumento } \\
\text { P11 }\end{array}$} & \multirow[t]{2}{*}{1} & \multirow[t]{2}{*}{2} & \multirow[t]{2}{*}{3} & \multirow[t]{2}{*}{4} & \multirow[t]{2}{*}{5} \\
\hline P11 & ¿Considera necesario publicar una investigación científica? & & & & & \\
\hline P12 & ¿Conoce los tipos de artículos publicables en una revista? & & & & & \\
\hline & Instrumento & 1 & 2 & 3 & 4 & 5 \\
\hline P13 & ¿Sus investigaciones son publicadas en Latindex, Scielo o Scopus? & & & & & \\
\hline P14 & $\begin{array}{l}\text { ¿Es necesario usar un software antiplagio antes de la edición de un } \\
\text { artículo científico? }\end{array}$ & & & & & \\
\hline P15 & ¿Reconoce el término "open Access"? & & & & & \\
\hline P16 & ¿Reconoce el término "evaluación por pares"? & & & & & \\
\hline P17 & ¿En su labor docente solicita que sus estudiantes investiguen? & & & & & \\
\hline P18 & $\begin{array}{l}\text { ¿Los trabajos realizados por los estudiantes durante el ciclo deberian } \\
\text { ser publicados en una revista científica? }\end{array}$ & & & & & \\
\hline P19 & En su labor docente ¿incentiva a la investigación de sus estudiantes? & & & & & \\
\hline P20 & $\begin{array}{l}\text { ¿Considera necesario que los estudiantes formen parte de un } \\
\text { "semillero de investigación"? }\end{array}$ & & & & & \\
\hline
\end{tabular}

\section{c) Competencias avanzadas}

\begin{tabular}{|c|c|c|c|c|c|c|}
\hline \multicolumn{2}{|r|}{ Instrumento } & 1 & 2 & 3 & 4 & 5 \\
\hline P21 & En elúltimo año ¿ha participado en una investigación multidisciplinaria? & & & & & \\
\hline P22 & $\begin{array}{l}\text { ¿Considera que el trabajo en equipo incrementaría la producción en } \\
\text { la universidad? }\end{array}$ & & & & & \\
\hline P23 & ¿Considera necesario realizar una investigación internacional? & & & & & \\
\hline
\end{tabular}

El instrumento utilizado fue validado por 5 expertos en la materia, los cuales aprobaron satisfactoriamente el instrumento referido; asimismo, se utilizó el software SPSS a fin de obtener el coeficiente de alfa de Cronbach, obteniéndose .966, resultado que según George y Mallery (2003) citados en FríasNavarro (2019) "sugiere la recomendación para evaluar los valores de los coeficientes de alfa de Cronbach", apreciándose que en el presente caso el resultado corresponde al Coeficiente alfa $>.9$, por lo tanto, es excelente.

\section{RESULTADOS Y DISCUSIÓN}

\section{Respecto a las competencias básicas}

La labor docente debe impulsar la creatividad e imaginación desde las aulas universitarias, para ello deberá compartir sus conocimientos relacionados (tabla 1) a la metodología de la investigación científica, redacción académica y estadística, representando un modelo a seguir en el proceso de formación del estudiante como futuro investigador. 
Tabla 1: Competencias investigativas básicas

\begin{tabular}{cccccc}
\hline & Nunca & Casi nunca & A veces & Casi siempre & Siempre \\
\hline P1 & $0 \%$ & $0 \%$ & $0 \%$ & $17 \%$ & $83 \%$ \\
P2 & $0 \%$ & $0 \%$ & $6 \%$ & $11 \%$ & $83 \%$ \\
P3 & $0 \%$ & $0 \%$ & $0 \%$ & $11 \%$ & $89 \%$ \\
P4 & $0 \%$ & $0 \%$ & $6 \%$ & $17 \%$ & $77 \%$ \\
P5 & $0 \%$ & $0 \%$ & $3 \%$ & $14 \%$ & $83 \%$ \\
P6 & $0 \%$ & $0 \%$ & $6 \%$ & $6 \%$ & $88 \%$ \\
P7 & $0 \%$ & $0 \%$ & $8 \%$ & $14 \%$ & $78 \%$ \\
P8 & $0 \%$ & $0 \%$ & $0 \%$ & $8 \%$ & $92 \%$ \\
P9 & $0 \%$ & $0 \%$ & $8 \%$ & $8 \%$ & $84 \%$ \\
P10 & $0 \%$ & $0 \%$ & $36 \%$ & $45 \%$ & $19 \%$ \\
\hline
\end{tabular}

En ese orden de ideas, surge como primera discusión, que el diseño metodológico debe ser la pieza principal en el proceso de investigación y como tal debe ser observada desde la fase previa a la identificación del problema a tratar, ello permitirá que el docente de forma propia perciba su actuación, calificándola de correcta o no en el ejercicio de su rol como docente, asesor, acompañante, revisor o jurado de tesis.

\section{Respecto a las competencias complementarias}

La investigación sin producción científica es un barco a la deriva; para ello se debe conocer la clasificación de artículos pasibles de ser publicados en una revista científica (tabla 2); aunque no exista un único camino para tal finalidad, el docente investigador deberá aprender de sus aciertos y desaciertos, siendo un ejemplo a seguir en sus estudiantes y un hito motivador para sus colegas del claustro universitario.

Tabla 2: Competencias investigativas complementarias

\begin{tabular}{cccccc}
\hline & Nunca & Casinunca & A veces & Casi siempre & Siempre \\
\hline P11 & $0 \%$ & $0 \%$ & $0 \%$ & $17 \%$ & $83 \%$ \\
P12 & $0 \%$ & $0 \%$ & $8 \%$ & $8 \%$ & $84 \%$ \\
P13 & $0 \%$ & $0 \%$ & $5 \%$ & $11 \%$ & $84 \%$ \\
P14 & $0 \%$ & $0 \%$ & $6 \%$ & $11 \%$ & $83 \%$ \\
P15 & $0 \%$ & $0 \%$ & $6 \%$ & $11 \%$ & $83 \%$ \\
P16 & $0 \%$ & $0 \%$ & $6 \%$ & $11 \%$ & $83 \%$ \\
P17 & $0 \%$ & $0 \%$ & $3 \%$ & $14 \%$ & $83 \%$ \\
P18 & $0 \%$ & $0 \%$ & $11 \%$ & $6 \%$ & $83 \%$ \\
P19 & $0 \%$ & $0 \%$ & $6 \%$ & $11 \%$ & $83 \%$ \\
P20 & $0 \%$ & $0 \%$ & $8 \%$ & $8 \%$ & $84 \%$ \\
\hline
\end{tabular}


De lo consignado, surge como segunda discusión ¿existe el deber de publicar"?, ante lo cual se debe entender que toda actividad producto de la investigación es necesaria su difusión, porque constituiría una alternativa u opción para resolver una situación problemática de la realidad, labor que debe ser promovida por el docente universitario en todas las asignaturas que tiene a su cargo.

\section{Respecto a las competencias avanzadas}

La investigación no debe ser segmentada y restringida al conocimiento de una determinada materia, es labor del docente unirse a grupos de investigación (tabla 3) nacionales $\mathrm{o}$ internacionales tendientes a intercambiar perspectivas de aprendizaje e identificación de soluciones a problemas comunes o cotidianos en la sociedad.

Tabla 3: Competencias investigativas avanzadas

\begin{tabular}{cccccc}
\hline & Nunca & Casinunca & A veces & Casi siempre & Siempre \\
\hline P21 & $78 \%$ & $11 \%$ & $11 \%$ & $0 \%$ & $0 \%$ \\
P22 & $0 \%$ & $0 \%$ & $0 \%$ & $17 \%$ & $83 \%$ \\
P23 & $0 \%$ & $0 \%$ & $0 \%$ & $19 \%$ & $81 \%$ \\
\hline
\end{tabular}

En ese sentido, surge como tercera discusión, que la investigación es multidisciplinaria y como tal el conocimiento no debe ser desplazado a una sola área del saber humano, por lo tanto, se debe reunir la información necesaria para brindar una solución a los problemas de la realidad.

\section{CONCLUSIONES}

El docente universitario se autopercibe como investigador, motivo por el cual deberá desarrollar competencias investigativas básicas, complementarias y avanzadas con la finalidad de ser protagonista en el proceso de formación de los estudiantes.

Las competencias investigativas básicas están relacionadas con los conocimientos derivados de la investigación científica, las complementarias se enfocan en la divulgación o difusión en revistas y las avanzadas centran su atención en el trabajo en equipo con una perspectiva multidisciplinaria.

La relación docente - estudiante, en el 2020, deberá estar encaminada a resolver los problemas de la realidad, no como una obligación sino como un deber inherente a la educación superior.

El fortalecimiento del rol docente se verifica en la medida que asume su participación activa e incentiva el desarrollo de las habilidades de los estudiantes en su proceso de formación como investigadores.

La investigación debe estar presente en cada asignatura de las carreras profesionales y técnicas, no es cuestión de sabiduría, al contrario, es la disciplina constate sustentada en una metodología con la finalidad de resolver un problema o comprobar una posible alternativa de solución.

\section{REFERENCIAS BIBLIOGRÁFICAS}

Aldana, J. (2017). La investigación social. Competencias en el docente universitario. Recuperado de http://bit.ly/39sq5AT

Araujo, M. (2016). Formación del Docente de Educación Media General desde el Enfoque por Competencias. Recuperado de http://bit.ly/2snFzp7 
Arrieta, W., Ocón, A., López, G., Buelvas, U. \& Monterroza, V. (2018). El desarrollo de la competencia investigativa en la formación de docentes. Caso: Programa Licenciatura en Matemáticas de la Universidad de Sucre. Recuperado de http://bit.ly/2MI9EXs

Aular, J., Marcano, N. \& Moronta, M. (2009). Competencias investigativas del docente de educación básica. Recuperado de http://bit.ly/2ZENQkM

Ayala, E. \& Barrera, J. (2018). Competencias investigativas en docentes universitarios. El caso del departamento de arquitectura de la Universidad Francisco de Paula Santander. Recuperado de http://bit. ly/2QtsdQ4

Balbo, J., Pacheco, M. \& Rangel, Z. (2015). Medición de las competencias investigativas en los docentes adscritos al departamento de ciencias sociales de la Universidad Nacional Experimental del Táchira. Recuperado de http://bit. ly/2tdhjWP

Benitorevollo, A., Olier, E., Medrano, Y. \& González, F. (2016). La práctica pedagógica y las competencias investigativas del docente egresado del programa en investigación aplicada a la educación de Cecar. Recuperado de http://bit.ly/39qEm0S

Borroto, E., Dueñas, F. \& Reyna, A. (2018). Identificación de necesidades de formación en competencias investigativas: herramienta para la implementación de planes de formación. Recuperado de http://bit.ly/2Mlw4I5

Buendía-Arias, X., Zambrano-Castillo, L. \& Alirio, E. (2018). El desarrollo de competencias investigativas de los docentes en formación en el contexto de la práctica pedagógica. Recuperado de http://bit. Iy/2ZAvNvW

Campos, J., Madriz, L., Brenes, O., Rivera, Y. \& Viales, M. (2012). Competencias investigativas en el personal académico de la Escuela de Ciencias de la Educación de la UNED, Costa Rica. Recuperado de http://bit.ly/36dkDj4

Cardoso, E. \& Cerecedo, M. (2018). Valoración de las Competencias Investigativas de los Estudiantes de Posgrado en Administración. Recuperado de http://bit. ly/2tfQCR9

Chacín, R. (2018). Competencias investigativas del docente universitario en tiempos postmodernos: aportes transteoréticos en el campo de las ciencias de la educación. Recuperado de http://bit.ly/37rS8yx

Cruzata-Martínez, A., Bellido, R., VelásquezTejeda, M. \& Alhuay-Quispe, J. (2018). La tutoría como estrategia pedagógica para el desarrollo de competencias de investigación en posgrado. Recuperado de http://bit.ly/39ro33T

Delgado, Y. \& Alfonzo, R. (2019). Competencias Investigativas del Docente Construidas durante la Formación Universitaria. Recuperado de http://bit.ly/2MJPIsm

Frías-Navarro, D. (2019). Apuntes de consistencia interna de las puntaciones de un instrumento de medida. Recuperado de http://bit.ly/2u62nKs

García-Gutiérrez, Z. \& Aznar-Díaz, I. (2019). E desarrollo de competencias investigativas, una alternativa para formar profesionales en pedagogía infantil como personal docente investigador. Recuperado de http://bit.ly/2Qc5s4n

García, J. (2019). Efectos del módulo 
autoinstructivo en las competencias investigativas de estudiantes de una universidad privada. Recuperado de http://bit.ly/2syYJZ5

Gayol, M., Monternegro, S., Tarrés, M. \& D'Octavio, A. (2008). Competencias investigativas. Su desarrollo en carreras del área de la Salud. Recuperado de http://bit.ly/37oVjac

Guity, S. \& Mendoza, J. (2018). Desarrollar estrategias didácticas para la adquisición de competencias investigativas en estudiantes de octavo grado del Centro de Investigación e Innovación Educativas. Recuperado de http://bit.ly/2rM9ffi

Marrero, O. \& Pérez, M. (2014). Competencias investigativas en la educación superior. Recuperado de http://bit.ly/2ZDvjoJ

Martínez, A., Patricia, M., Calderón, N., Corredor, N., Alfaro, J., Llerena, A., Benavides, G., Garzón, P., Escorcia, J. \& Henríquez, H. (2015). La formación del docente investigador. Un estudio en las facultades de Educación del Caribe Colombiano. Recuperado de http://bit.ly/2u0T6Di

Mas, O. (2011). El profesor universitario: sus competencias y formación. Recuperado de http://bit.ly/2F7QDti

Nakamura, P., Rivero, C. \& Velasco, A. (2019). Desarrollando competencias investigativas en los estudiantes de educación a través del aprendizaje situado. Recuperado de http://bit. ly/36nJZL6

Ollarves, Y. \& Salguero, L. (2009). Una propuesta de competencias investigativas para los docentes universitarios. Recuperado de http://bit.ly/37op8re
Parra, L., Marsollier, R. \& Difabio, H. (2019). Diferencias y relaciones en las habilidades y competencias de los licenciados en Colombia. Recuperado de http://bit. ly/35gKZja

Paz, C., Estrada, L. Chinchilla, B. \& Valladares, N. (2017). Desarrollo de Competencias Investigativas en Pregrado. Recuperado de http://bit.ly/356sttr

Paz, L. \& Fierro, Y. (2015). Competencias investigativas en los docentes beneficiados por la estrategia de formación y acceso para la apropiación pedagógica de las TIC. Recuperado de http://bit.ly/2ZAwbKX

Pérez, M. (2012). Fortalecimiento de las competencias investigativas en el contexto de la educación superior en Colombia. Recuperado de http://bit.ly/2snGGFj

Ponce, D., Pupo, A., Ponce, A., Viteri, D., \& Álvarez, L. (2017). La formación de Competencias Investigativas y su Transcendencia en el Liderazgo Educativo. Propuesta Metodológica. Recuperado de http://bit.ly/2tYNhpU

Pulido, J. (2017). Competencias Investigativas de los aspirantes a ingresar al programa de Postgrado de la UPEL-IMPM. Recuperado de http://bit.ly/2SQebLI

Reiban, R. (2018). Las competencias investigativas del docente universitario. Recuperado de http://bit.ly/37npE8T

Reiban, R., De la Rosa, H. \& Zeballos, J. (2017). Competencias investigativas en la Educación Superior. Recuperado de http://bit.ly/2u72iGn

Reiban, R. \& Vera, V. (2017). Análisis de la producción científica: Caso docentes de Nivelación de la Universidad de Guayaquil. Recuperado de http://bit.ly/2SLWBYu 
Ricardo, M., Amat, M., Andrade, D., Jiménez, R. \& Cisneros, C. (2019). Desarrollo de competencias investigativas formativas: retos y perspectivas para la Universidad. Recuperado de http://bit.ly/2QuCPhA

Rivera, E. (2017). La investigación como una competencia necesaria en la práctica docente durante la formación inicial. Recuperado de http://bit.ly/39tzfgb

Rojas, C. \& Aguirre, S. (2015). La formación investigativa en la educación superior en América Latina y El Caribe: una aproximación a su estado del arte. Recuperado de http://bit.ly/2ZBzykA

Rubio, M., Torrado, M., Quirós, C. \& Valls, R. (2018). Autopercepción de las competencias investigativas en estudiantes de último curso de Pedagogía de la Universidad de Barcelona para desarrollar su Trabajo de Fin de Grado. Recuperado de http://bit.ly/2Q9r29q

Tapia, C., Cardona, S. \& Vázquez, H. (2018). Las competencias investigativas en posgrado: experiencia de un curso en línea. Recuperado de http://bit.ly/2Q8KCCP

Toro, I., Saldarriaga, J., León, M., Martínez, J. \& Arias, O. (2014). Competencias docentes para la enseñanza de la metodología de la investigación y la evaluación de trabajos de grado y tesis doctorales en Administración. Recuperado de http://bit. ly/2SGMDHW

Tovar-Gálvez, J. (2018). Formación investigativa de profesores: el problema como producto del proceso de problematización. Recuperado de http://bit.ly/2tYNSrE

Véliz, A. \& Lovato, S. (2019). Las competencias investigativas en docentes. Recuperado de http://bit.ly/2QzqPeB
Veytia, M. (2012). Desarrollo de competencias investigativas en estudiantes de doctorado a partir del uso de las tecnologías de la información y la comunicación. Recuperado de http://bit.ly/2F3TbZI

Zúñiga, X. \& Pando, T. (2019). Las competencias investigativas y el aprendizaje basado en problemas. Un estudio cuasiexperimental. Recuperado de http://bit.ly/2QxtW75 